\title{
Author Correction: A strong, biodegradable and recyclable lignocellulosic bioplastic
}

Qinqin Xia®D, Chaoji Chen (D), Yonggang Yao, Jianguo Li, Shuaiming He, Yubing Zhou, Teng Li (D), Xuejun Pan (D), Yuan Yao (iD and Liangbing Hu (D)

Correction to: Nature Sustainability https://doi.org/10.1038/s41893-021-00702-w, published online 25 March 2021.

In the version of this Article initially published, there were labeling errors in Fig. 3k and Extended Data Fig. 6. In Fig. 3k, the original $y$-axis label " $2 \theta\left({ }^{\circ}\right)$ " and $x$-axis label "Intensity (a.u.)" appeared on the wrong axes and are now swapped with each other. In Extended Data Fig. 6, top left panel, the label "Lignicell plastic" has been changed to read "Lignocellulosic bioplastic" and the label "Polystyrene" has been changed to read "PVC."

These changes have been made in the online version of the Article.

Published online: 20 August 2021

https://doi.org/10.1038/s41893-021-00771-x

(C) The Author(s), under exclusive licence to Springer Nature Limited 2021 